\section{T time for tumor vasculature}

\section{By Chris Cain, Staff Writer}

A National Cancer Institute study has shown that T cells engineered to seek out vascular endothelial growth factor receptor 2 can stop tumor growth in mice. ${ }^{1} \mathrm{NCI}$ has advanced the engineered cells into a Phase I/II dose-escalation trial to treat metastatic cancer, although the complexity of the technique could make it difficult to commercialize.

For more than 20 years, Steven Rosenberg, chief of surgery at NCI, has been working on adoptive transfer of $\mathrm{T}$ cells to treat cancer. In a 1988 paper in The New England Journal of Medicine, he first reported that tumor-reactive $\mathrm{T}$ cells could be isolated from patients with metastatic melanoma, cultured ex vivo and reintroduced into patients to induce cancer regression. $^{2}$

In a 2006 Science paper, Rosenberg reported a key advance in the technique: instead of isolating a patient's tumor-reactive T cells,

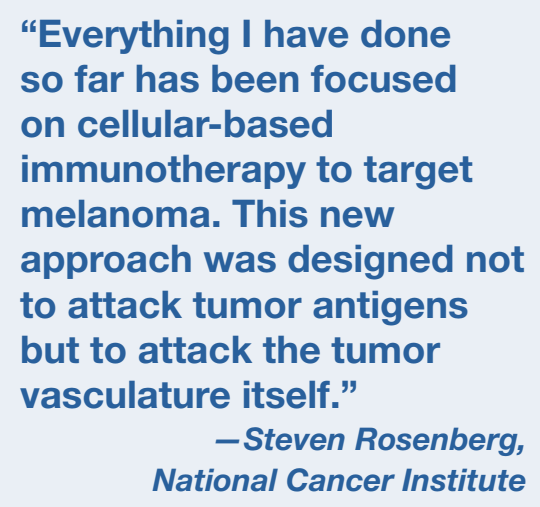
so far has been focused on cellular-based immunotherapy to target melanoma. This new approach was designed not to attack tumor antigens but to attack the tumor vasculature itself." -Steven Rosenberg, National Cancer Institute

In mice with solid tumors originating from five different cancer cell lines, the engineered $\mathrm{T}$ cells inhibited tumor growth and extended survival compared with control T cells.

The researchers also engineered a chimeric antigen receptor specific for VEGFR-2. Primary human T cells transduced with this receptor were able to selectively kill VEGFR-2+ cells and leave VEGFR-2- cells untouched, suggesting this technique could be used to treat cancer in humans.

This month, NCI started a Phase I/II trial of the engineered cells in patients with refractory metastatic cancer (see Figure 1, "Fighting cancer with VEGFR-2-targeting T cells").

"This trial is like nothing that has been done before" because of the breadth of cancer types that could be treated with the approach, said Rosenberg.

Laurence Cooper, associate professor and section chief of pediatric cell therapy at The University of Texas M.D. Anderson Cancer Center, agreed. "This study presents an important advance because it allows investigators to go beyond tailoring cells to a specific patient's tumor type. It allows one to fashion cells to a particular pathology, namely the tumor environment, which is common to tumors occurring in many patients," he said.

Cooper's lab is focused on treating lymphomas and leukemias with adoptive $\mathrm{T}$ cell therapy. Last year, he founded Incellerate LLC, which is using electroporation to transfer chimeric antigen receptors into $\mathrm{T}$ cells without he engineered the patient's $\mathrm{T}$ cells to target a melanoma antigen of choice. ${ }^{3}$ This removed the need for a patient to already carry reactive $\mathrm{T}$ cells for a chosen antigen and allowed researchers to engineer $\mathrm{T}$ cells to recognize virtually any target.

Rosenberg hypothesized that the approach could be extended to a wide range of tumors using a universal cancer antigen. He settled on vascular endothelial growth factor receptor 2 (KDR/Flk-1; VEGFR-2), a tyrosine kinase that is overexpressed in tumor endothelial cells. At least 17 companies have VEGFR-2 inhibitors in development.

"Everything I have done so far has been focused on cellular-based immunotherapy to target melanoma. This new approach was designed not to attack tumor antigens but to attack the tumor vasculature itself," Rosenberg told SciBX.

In a new paper published in The Journal of Clinical Investigation, Rosenberg's group describes the design of a chimeric antigen receptor that could specifically target Flk-1, which is the mouse version of VEGFR-2. The product is a fusion protein that contains the antigenbinding fragment of an antibody linked to protein domains that are known to signal $\mathrm{T}$ cell activation upon target binding.

Primary mouse T cells were engineered to express the receptor by retroviral transduction. In cell culture, the engineered $\mathrm{T}$ cells selectively killed Flk-1 $1^{+}$cells and had no effect on Flk-1- control cells. the use of viral vectors. The company is in its infancy and is searching for a management team.

\section{Adopting an approach}

Almost all clinical trials of adoptive immunotherapies using genetically modified $\mathrm{T}$ cells to treat cancer have been single-institution Phase I/II studies. "In the absence of a unified structure, everyone has grown cell therapy according to their best efforts at their own institutions, so it's all quite fragmented," said Cooper. "One investigator's approach to T cell therapy is not the same as another person's cell therapy, and without multicenter trials it is difficult to establish efficacy."

The exception is Dendreon Corp., which markets Provenge sipuleucel-T to treat metastatic prostate cancer. With Provenge, a patient's white blood cells are collected, shipped to a Dendreon facility, exposed to a tumor antigen and then shipped back to be reinfused into the patient.

Rosenberg thinks Dendreon's model is the most likely way the technique reported in the JCI paper could be commercialized.

Cooper said an alternative approach for translating Rosenberg's technology would be to mirror the way bone marrow transplantation is currently handled. Transplants occur as a hospital-based service 


\section{ANALYSIS}

\section{TARGETS \& MECHANISMS}

Figure 1. Fighting cancer with VEGFR-2-targeting T cells. A Phase I/II clinical trial being conducted by the National Cancer Institute (http://bethesdatrials.cancer.gov/clinicalresearch/search_detail.aspx?ProtocollD=NCl-11-C-0013) is seeking to test the hypothesis that engineered T cells could help treat metastatic cancer. White blood cells are isolated from patients with metastatic cancer refractory to at least one prior treatment [a], expanded ex vivo and transduced with a retroviral vector carrying a chimeric antigen receptor recognizing vascular endothelial growth factor receptor 2 (KDR/Flk-1; VEGFR-2) [b].

The chimeric antigen receptor is a fusion protein consisting of the VEGFR-2 binding fragment of an antibody linked to protein domains that signal $\mathrm{T}$ cell activation upon target binding.

Finally, patients receive nonmyeloablative, lymphocytedepleting therapy and are injected with the modified T cells bearing the chimeric receptor. IL-2 is administered for up to five days after treatment to keep modified cells active [c].

that relies on local expertise and processing, and doctors and staff are compensated for the treatment they provide.

Manufacture of the engineered $\mathrm{T}$ cells could involve multiple federally funded centers, analogous to blood banks that collect and process cells according to a common set of rules. Trained doctors would handle the complex task of managing the treatment, which includes chemotherapy, to deplete the patient's immune system, and IL-2, a cytokine that activates the newly transferred cells.

“The NCI's technology is really promising, and it needs support and further testing, which may need to come from the nonprofit sector and the government," said Cooper.

In addition to the Phase I/II trial, Rosenberg has submitted an IND to the FDA for a new study that uses gene-modified lymphocytes to target melanoma-associated antigen A3 (MAGEA3), an antigen found on many common cancers.

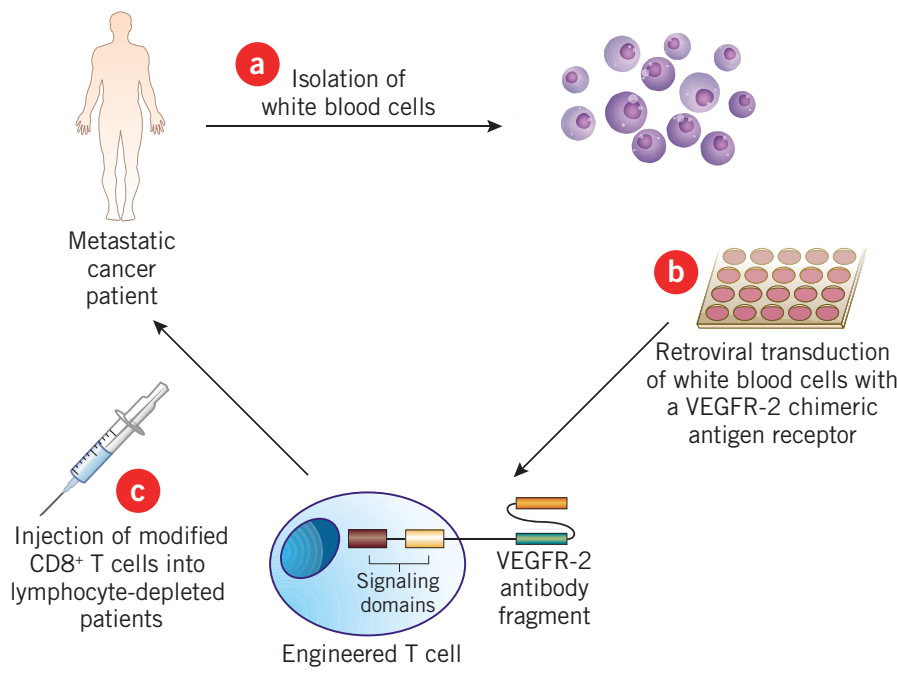

NCI has filed for patents covering the technology described by Rosenberg, and the work is available for licensing.

Cain, C. SciBX 3(45); doi:10.1038/scibx.2010.1344

Published online Nov. 18, 2010

REFERENCES

1. Chinnasamy, D. et al. J. Clin. Invest.; published online Oct. 11, 2010; doi: $10.1172 / \mathrm{JCl} 43490$

Contact: Steven A. Rosenberg, National Cancer Institute,

Bethesda, Md.

e-mail: SAR@mail.nih.gov

2. Rosenberg, S.A. et al. N. Engl. J. Med. 319, 1676-1680 (1988)

3. Morgan, R.A. et al. Science 314, 126-129 (2006)

COMPANIES AND INSTITUTIONS MENTIONED

Dendreon Corp. (NASDAQ:DNDN), Seattle, Wash.

Incellerate LLC, Houston, Texas

National Cancer Institute, Bethesda, Md.

The University of Texas M.D. Anderson Cancer Center,

Houston, Texas 